\title{
Perspective: Application of the American College of Medical Genetics variant interpretation criteria to Common Variable Immunodeficiency Disorders
}

\author{
Rohan Ameratunga ${ }^{1}$, Caroline Allan ${ }^{1}$, Klaus Lehnert $^{2}$, and See-Tarn Woon ${ }^{3}$ \\ ${ }^{1}$ Auckland Hospital \\ ${ }^{2}$ University of Auckland \\ ${ }^{3}$ Auckland City Hospital
}

May 5, 2020

\begin{abstract}
Common Variable Immunodeficiency Disorders (CVIDs) are rare Primary Immunodeficiency diseases (PIDs) associated with late onset antibody failure leading to immune system failure. Most patients suffer recurrent and severe infections, while some have predominantly autoimmune and inflammatory disorders. In recent years a large number of genetic defects have become associated with these disorders. Next Generation Sequencing (NGS) allows the analysis of multiple genes simultaneously. The mutations identified from NGS are evaluated with the American College of Medical Genetics (ACMG) variant interpretation criteria to determine their pathogenicity (causality). We have advocated all patients with a CVID phenotype should undergo genetic testing but acknowledge the complexity of the genetics associated with these disorders. Variants of Unknown Significance (VUS) remain a significant barrier to realising the full potential of NGS in CVID and CVID-like disorders. We review the nuances of the application of the ACMG variant interpretation criteria to patients with a CVID phenotype.
\end{abstract}

\section{Introduction}

Common Variable Immunodeficiency disorders (CVIDs) are the most frequent primary immunodeficiency disorder (PID) in adults. Patients with CVID suffer late onset antibody failure leading to immune system failure.[1]

Current estimates suggest a prevalence between 1:25 000 to 1:50 000 in Caucasians. [2] For reasons that are not clear these disorders appear to be less prevalent in Asian and African populations, although there may be ascertainment bias. [3]

Most patients with CVID suffer recurrent and severe infections, often leading to chronic disabling sequelae including bronchiectasis and chronic upper respiratory tract suppuration. [4] A substantial minority of patients experience predominantly autoimmune and inflammatory disorders consequent to immune dysregulation. [5, 6] Some of these patients have severe hypogammaglobulinemia with minimal numbers of infections.

The advent of subcutaneous and intravenous immunoglobulin (SCIG/IVIG) replacement has resulted in substantial improvement in morbidity and mortality in patients with CVID.

Over the last two decades there have been major advances in the understanding of this rare group of disorders.[7] These have included the spectrum of phenotypic features of well-characterised cohorts from around the world as well as better understanding of the genetic basis of CVID. In parallel with these developments there have been refinement and validation of diagnostic criteria for CVID. [8-10] All of these advances have improved the understanding of this rare group of enigmatic conditions. 
In recent years, a large number of genetic variants have been identified in association with CVID. In 2003, the first genetic defect was identified in the Black Forest area of Germany, causing a combined immunodeficiency. [11] All individuals carried the identical mutation in ICOS indicating a founder population. Mutations of the ICOS gene have subsequently been identified in other parts of the world, confirming the existence of allelic heterogeneity.[12]

Shortly afterwards a defect of TACI (TNFRSF13B ) was identified.[13] Although TACI mutations are found in higher numbers of CVID patients than in the general population, their prevalence in the community far exceeds the prevalence of CVID. [14] We have shown that TACI mutations predispose to and modify disease severity in CVID. [15, 16] Variants of other genes including BAFFR (TNFRSF13C), TWEAK (TNFSF12 ), MSH5 and TRAIL (TNFSF10) similarly predispose to or modify disease severity.

All current definitions of CVID exclude known causes of hypogammaglobulinemia. [17-19] We have thus termed patients with causative genetic defects (NFKB1, NFKB2 etc.) as having CVID-like disorders, while those with variants predisposing to or modifying the condition remain within the spectrum of CVID. [9]

CVID-like disorders exhibit marked locus heterogeneity (genocopy). [5] Thus, mutations of several genes can present with recurrent and severe infections or with inflammatory and autoimmune disorders. Because there are a large number of genetic variants associated with CVID, it can be difficult to identify the genetic defect likely responsible for the condition on clinical features alone. [20] Sometimes the presence of a specific clinical feature such as alopecia could indicate a mutation of the NFKB1 or NFKB2 genes. [21] In most cases however, such clues are absent. This locus heterogeneity is a strong argument for routine sequencing of all genes associated with CVID and CVID-like disorders.[5]

Genetic sequencing of patients with a CVID phenotype has now become feasible with the advent of NGS. Serial Sanger sequencing of 40 or more individual genes associated with CVID and CVID-like disorders was not an effective use of valuable resources. [20] NGS allows the parallel and cost-effective sequencing of a large number of genes, which had a major impact on the diagnosis of CVID and CVID-like disorders. It now appears over $25 \%$ of patients from non-consanguineous populations have an underlying genetic defect. [22] In consanguineous populations, the proportion is much higher. [20] In consanguineous populations, these are predominantly autosomal recessive disorders, but unexpectedly, many have autosomal dominant disorders, presumably from de novo mutations.[20]

Identification of the genetic basis of PIDs has profound implications for the patient and their family (Table 1). $[23,24]$ It allows certainty of diagnosis, family studies, as well as reproductive options including preimplantation genetic diagnosis and chorionic villus sampling. Importantly it has ushered in the era of precision medicine where treatments can now be targeted to individual genetic defects. Examples include the use of mTOR inhibitors in PIK3CD andPIK3R1 defects and abatacept in CTLA-4 or LRBAdefects. This is in addition to SCIG/IVIG for the hypogammaglobulinemia.

NGS for CVID can be divided into two approaches. The first is diagnostic and the second is for gene discovery research. While NGS has had a major impact on the identification of CVID-like disorders, it has also complicated the genetic assessment of many patients. Variants of unknown significance (VUS) have emerged as a major factor hindering diagnostic studies of CVID and CVID-like disorders.

The American College of Clinical Genetics (ACMG) has published several iterations of their guidelines for the interpretation of genetic variants. [25] Given their genetic, allelic and phenotypic heterogeneity, CVID and CVID-like disorders pose a significant challenge for implementation of these ACMG variant interpretation guidelines. In this article, we review the complexities of the application of ACMG guidelines for the interpretation of variants identified from NGS protocols in patients with a CVID phenotype.

Description of the ACMG criteria .

\begin{tabular}{llllll}
\hline Benign & Benign & Pathogenic & Pathogenic & Pathogenic & Pathogenic \\
\hline Strong & Supporting & Supporting & Moderate & Strong & Very strong
\end{tabular}




\begin{tabular}{|c|c|c|c|c|c|c|}
\hline & Benign & Benign & Pathogenic & Pathogenic & Pathogenic & Pathogenic \\
\hline $\begin{array}{l}\text { Population } \\
\text { data }\end{array}$ & $\begin{array}{l}\text { MAF is too } \\
\text { high for } \\
\text { disorder } \\
\text { BA1/BS1 } \\
\text { OR } \\
\text { observation } \\
\text { in controls } \\
\text { inconsistent } \\
\text { with disease } \\
\text { penetrance } \\
\text { BS2 }\end{array}$ & & & $\begin{array}{l}\text { Absent in } \\
\text { population } \\
\text { databases } \\
\text { PM2 }\end{array}$ & $\begin{array}{l}\text { Prevalence } \\
\text { in affecteds } \\
\text { statistically } \\
\text { increased } \\
\text { over controls } \\
\text { PS4 }\end{array}$ & \\
\hline $\begin{array}{l}\text { Computational } \\
\text { and predictive } \\
\text { data }\end{array}$ & & $\begin{array}{l}\text { Multiple lines } \\
\text { of } \\
\text { computational } \\
\text { evidence } \\
\text { suggest no } \\
\text { impact on } \\
\text { gene/gene } \\
\text { product BP4 } \\
\text { Missense in } \\
\text { gene where } \\
\text { only } \\
\text { truncating } \\
\text { cause disease } \\
\text { BP1 Silent } \\
\text { variant with } \\
\text { non-predicted } \\
\text { splice impact } \\
\text { BP7 In-frame } \\
\text { indels in } \\
\text { repeat w/out } \\
\text { known } \\
\text { function BP3 }\end{array}$ & $\begin{array}{l}\text { Multiple lines } \\
\text { of } \\
\text { computational } \\
\text { evidence } \\
\text { support a } \\
\text { deleterious } \\
\text { effect on the } \\
\text { gene/gene } \\
\text { product PP3 }\end{array}$ & $\begin{array}{l}\text { Novel missense } \\
\text { change at an } \\
\text { amino acid } \\
\text { residue where } \\
\text { a different } \\
\text { pathogenic } \\
\text { missense } \\
\text { change has } \\
\text { been seen } \\
\text { before PM5 } \\
\text { Protein length } \\
\text { changing } \\
\text { variant PM4 }\end{array}$ & $\begin{array}{l}\text { Same amino } \\
\text { acid change as } \\
\text { an established } \\
\text { pathogenic } \\
\text { variant PS1 }\end{array}$ & $\begin{array}{l}\text { Predicted null } \\
\text { variant in a } \\
\text { gene where } \\
\text { LOF is a } \\
\text { known } \\
\text { mechanism of } \\
\text { disease PVS1 }\end{array}$ \\
\hline $\begin{array}{l}\text { Functional } \\
\text { data }\end{array}$ & $\begin{array}{l}\text { Well- } \\
\text { established } \\
\text { functional } \\
\text { studies show } \\
\text { no } \\
\text { deleterious } \\
\text { effect BS3 }\end{array}$ & & $\begin{array}{l}\text { Missense in } \\
\text { gene with } \\
\text { low rate of } \\
\text { benign } \\
\text { missense } \\
\text { variants and } \\
\text { path. } \\
\text { missenses } \\
\text { common } \\
\text { PP2 }\end{array}$ & $\begin{array}{l}\text { Mutational } \\
\text { hot spot or } \\
\text { well-studied } \\
\text { functional } \\
\text { domain } \\
\text { without } \\
\text { benign } \\
\text { variation } \\
\text { PM1 }\end{array}$ & $\begin{array}{l}\text { Well- } \\
\text { established } \\
\text { functional } \\
\text { studies show } \\
\text { a deleterious } \\
\text { effect PS3 }\end{array}$ & \\
\hline
\end{tabular}




\begin{tabular}{|c|c|c|c|c|c|c|}
\hline & Benign & Benign & Pathogenic & Pathogenic & Pathogenic & Pathogenic \\
\hline $\begin{array}{l}\text { Segregation } \\
\text { data }\end{array}$ & $\begin{array}{l}\text { Non- } \\
\text { segregation } \\
\text { with disease } \\
\text { BS4 }\end{array}$ & & $\begin{array}{l}\text { Co- } \\
\text { segregation } \\
\text { with disease } \\
\text { in multiple } \\
\text { affected } \\
\text { family } \\
\text { members } \\
\text { PP1 }\end{array}$ & $\begin{array}{l}\text { Increased } \\
\text { segregation } \\
\text { data }\end{array}$ & & \\
\hline $\begin{array}{l}\text { De novo } \\
\text { data }\end{array}$ & & & & $\begin{array}{l}\text { De novo } \\
\text { (without } \\
\text { paternity } \\
\text { and } \\
\text { maternity } \\
\text { confirmed) } \\
\text { PM6 }\end{array}$ & $\begin{array}{l}\text { De novo } \\
\text { (paternity } \\
\text { and } \\
\text { maternity } \\
\text { confirmed) } \\
\text { PS2 }\end{array}$ & \\
\hline Allelic data & & $\begin{array}{l}\text { Observed in } \\
\text { trans with a } \\
\text { dominant } \\
\text { variant BP2 } \\
\text { Observed in } \\
\text { cis with a } \\
\text { pathogenic } \\
\text { variant BP2 }\end{array}$ & & $\begin{array}{l}\text { For recessive } \\
\text { disorders, } \\
\text { detected in } \\
\text { trans with a } \\
\text { pathogenic } \\
\text { variant PM3 }\end{array}$ & & \\
\hline $\begin{array}{l}\text { Other } \\
\text { database }\end{array}$ & & $\begin{array}{l}\text { Reputable } \\
\text { source } \\
\text { w/out } \\
\text { shared } \\
\text { data= } \\
\text { benign BP6 }\end{array}$ & $\begin{array}{l}\text { Reputable } \\
\text { source= } \\
\text { pathogenic } \\
\text { PP5 }\end{array}$ & & & \\
\hline Other data & & $\begin{array}{l}\text { Found in } \\
\text { case with an } \\
\text { alternate } \\
\text { cause BP5 }\end{array}$ & $\begin{array}{l}\text { Patient's } \\
\text { phenotype } \\
\text { of FH highly } \\
\text { specific for } \\
\text { gene PP4 }\end{array}$ & & & \\
\hline
\end{tabular}

Figure 1. The ACMG guidelines for the interpretation of variants following NGS.[25]

The ACMG criteria aim to determine the pathogenic significance (causality) of a variant in an individual patient. The ACMG criteria define six categories from very strongly pathogenic to strongly benign. Between likely benign and likely pathogenic are Variants of Unknown Significance (VUS), usually where there is contradictory in silico evidence or where there is insufficient data to reach a conclusion. As we discuss below, close collaboration with genetics professionals can play a crucial role in resolving many VUS.

The top row of the ACMG criteria compares the frequency of the disease associated variant with that in the community. GnomAD and ExAC are two common databases which are often consulted when determining variant allele frequency in "healthy" populations. In general, a variant present in more than $5 \%$ of the population is regarded as benign. CVID-like disorders are extremely rare with frequencies in the order of $0.01 \%$ or less. If variants have a high allele specific frequency in the healthy population, it is unlikely these underlie the pathogenicity of the condition. Exceptions include founder populations and common ethnic specific variants such as sickle cell disease. In the case of CVID and CVID-like disorders, variants of TACI 
(TNFRSF13B ), BAFFR (TNFRSF13C), TWEAK (TNFSF12), MSH5 and TRAIL (TNFSF10) are unlikely to be causative as their allele frequency far exceeds that of CVID. CVID cohorts have higher rates of these variants than the wider healthy population, indicating these are likely to predispose to or modify these disorders.[16]

It is important to note that variant frequencies for specific ethnicities are missing in large databases. What may seem to be a pathogenic mutation may simply be a common polymorphism in an under-surveyed ethnic group. Furthermore, databases from healthy individuals may contain a disproportionate number of heterozygotes compared to the Hardy-Weinberg principle, suggesting homozygosity is pathogenic. Homozygous individuals may either be deceased or too ill to be part of a healthy database. Such homozygous variants in patients with a CVID phenotype will need to be interpreted with caution. [5]

The second row of the ACMG criteria deals with in silico analysis of the variant. There are several computer algorithms, which attempt to predict the in vivo consequences of the variant. Some programmes determine whether the mutation reduces the half-life of mRNA, alters a critical element of the protein such as the catalytic function in the case of an enzyme, the ligand-binding domain of a receptor or it's correct subcellular localisation. Mutations at highly conserved and therefore functionally important sites can be identified by interspecies comparison as a surrogate marker of evolutionary conservation. Computer programmes can sometimes lead to contradictory results as each has strengths and weaknesses. There has been a move to replace individual predictions with those generated by meta-analysis tools such as REVEL, where an aggregate score is generated from the results of all of these programmes. This is analogous to the Forest plots in Cochrane meta-analysis data. This may offer a more reliable assessment of the pathogenicity of a variant.[26]

The third row examines the functional effects of the variant in vitro. A valid in vitro assay should closely reflect the function of the protein in vivo. The functional assay can be based on isolated cells or in a cell free system. The in vitro assay should reliably and reproducibly reflect the consequences of the variant compared to a wild type control. In routine diagnostic laboratories, such functional assays may not be available for all variants. Sending samples to research laboratories may be problematic given the stringent quality controls required for accreditation of diagnostic laboratories.

The fourth row examines familial segregation data. This requires access to other members of the family after genetic counselling. It would be expected that the variant will be present in symptomatic members of the family but not those who are unaffected. Co-segregation of variant with the phenotype offers powerful evidence of causality. Conversely, if the variant does not segregate with disorder, this is strong evidence for non-pathogenicity. The variable penetrance and expressivity in the case of CVID-like disorders are important caveats as discussed below.

The fifth row examines whether the mutation has arisen de novo. The presence of a de novo mutation in affected individuals is powerful evidence of causality, assuming paternity is not in dispute and parents with wild type sequence are clinically unaffected.

Allelic data comprise the sixth row of the ACMG criteria. These include whether the variant in question is in cis or trans with a known pathogenic variant. This is important in determining if the novel variant could be contributing to compound heterozygosity. A variant in cis with a known mutation may not be pathogenic but might contribute to disease if it is in trans. Determining the phase of such variants can be challenging for routine diagnostic laboratories as it may require access to techniques such as long-range PCR or cloning.

The seventh line describes the presence of the variant in another database, typically collections of diseasespecific variants curated by experts. The eighth row is determined by expert opinion on specific databases.

Although these criteria were developed for assessing the pathogenicity of new variants in genes known to cause disease, they are frequently applied to confirm the pathogenicity of mutations in genes not previously implicated in the patient's disease. This will pose similar challenges for determining the pathogenicity of novel CVID-like genes also. 


\section{Discussion: The genetic and phenotypic challenges posed by CVID and CVID-like disorders}

CVID and CVID-like disorders present a special challenge to variant interpretation by the ACMG criteria (Table 2). In addition to a large number of implicated genes, there are marked variations in the penetrance and expressivity of most variants associated with CVID and CVID-like disorders. [27] This may obfuscate the significance of individual variants on disease causality.

It is also clear that CVID and CVID-like disorders can present for the first time in seventh or eighth decades. It is possible environmental triggers such as Herpes virus infections trigger symptomatic disease and alter the prognostic trajectory of such patients. This is analogous to patients with X-linked Lymphoproliferative Disease (XLP), who remain well until suffering a catastrophic EBV infection. [28] The potential late onset of disease poses significant challenges for segregation studies or determining de novo pathogenicity. Late onset must also be taken into account when assessing the population frequency of a candidate variant; ideally, the variant frequency is obtained from population subsets of similar or higher age.

CVID-like disorders are also characterised by great phenotypic heterogeneity. We and others have shown marked differences in phenotype within the same family, carrying the identical mutation. In the family where we co-discovered the NFKB1 (465dupA) mutation, the disease severity ranged from an asymptomatic brother, with an affected child, to a sister who died from late onset combined immunodeficiency. [21, 29] Another brother had the autoimmune variant and the affected mother had mild immune thrombocytopenia. This phenotypic heterogeneity led to the erroneous initial discounting of the pathogenic significance of the NFKB1 variant, during trio-analysis for gene discovery. Reassignment of the correct phenotype resulted in identification of the causal NFKB1 mutation. Thus, clinically unaffected relatives can be carriers for the mutation, but pass the mutation to children who experience symptomatic disease at an early age. Similar phenotypic heterogeneity has been observed in other families carrying different mutations of the NFKB1 as well as mutations of other genes such $A D A 2$ deficiency, which cause CVID-like disorders.

CVID is genetically complex. There are suggestions that epigenetic changes could account for some cases of CVID. Identical twin brothers discordant for CVID were shown have differing DNA methylation patterns for several genes in including TCF3. [30] The stability of these changes will need to be monitored over time, to confirm epigenetic variations cause CVID-like disorders. The ACMG criteria cannot be applied to situations where epigenetic changes are thought to be the basis of the disorder (Table 2).

It is likely many patients with CVID and CVID-like disorders have oligogenic disease. We have shown the existence of a digenic disorder leading to quantitative epistasis in a patient with a CVID-like disorder. [16] Epistasis is the synergistic, non-linear interaction of two or more genetic loci resulting in either a much more severe disorder or an entirely different phenotype. We have termed these quantitative and qualitative epistasis. [31] The family we studied included most permutations and combinations of the TACI(c.310T $>$ C, C104R) and TCF3(T168fsX191) mutations. The proband who was worst affected had mutations of both TACI and TCF3 . Other family members carrying only one mutation were less severely affected.

In our family the TCF3 gene product (E2A) is a key transcription factor for many genes including immunoglobulin isotype switching and secretion. TACI plays a key role in immunoglobulin isotype switching and lies in tandem with genetic products transcribed by E2A. This synergistic nonlinear interaction caused genetic, biochemical and clinical (phenotypic) epistasis leading to a severe CVID-like disorder in the proband. $[16]$

This family illustrates some of the difficulties in applying the ACMG criteria to patients with digenic or oligogenic disorders leading to CVID-like conditions. The presence of variants predisposing to or modifying disease severity will complicate application of the ACMG guidelines to CVID-like disorders. We suggest ACMG guidelines should not be applied to mutations of genes such as TACI (TNFRSF13B), BAFFR (TNFRSF13C), TWEAK (TNFSF12), MSH5 and TRAIL (TNFSF10). They should be considered modifying or predisposing mutations rather than VUS, in the ACMG guidelines. No therapeutic decisions should be made purely on the presence of such predisposing variants alone. The use of the TACI mutation in reproductive decisions could have resulted in an adverse outcome, as it was the subsequently discovered 
TCF3 mutation, which was pathogenic in this family.

We have previously discussed the complexities in attributing causality in patients suffering from digenic disorders. [31] The ACMG has stated their guidelines should only be applied to causative monogenic disorders. [25] Our experience would support these recommendations. We do however feel the ACMG guidelines could be applied to the dominant mutation in digenic disorders, based on phenotypic assessment of the kindred. We have termed the dominant mutation the epistatic hub. [31] Such dominant mutations in epistatic hubs are likely to affect a critical molecule such as a cell surface receptor or a transcription factor affecting many downstream genes.

In this family, the TCF3 mutation appears to be highly penetrant and seems to behave in manner akin to $B T K$. The ACMG criteria could be confidently used for this mutation. Mutations in other genes leading to CVID-like disorders are more complex and can cause considerable uncertainty in terms of an individual patient's prognostic trajectory. As illustrated in our family with the NFKB1 mutation, one affected brother remains in excellent health and has normal immunoglobulins, although his daughter is severely affected.

The unpredictability caused by variable penetrance and expressivity in CVID-like disorders will make it very difficult to determine which patients should undergo bone marrow transplantation. In contrast to autosomal recessive $L R B A$ deficiency, a substantial proportion of patients with $C T L A-4$ mutations causing haploinsufficiency are asymptomatic. Such mutations will pose a dilemma for patients wishing to undergo chronic villus sampling to terminate a pregnancy. All of these nuances will need to be carefully explored with the patient during genetic counselling, prior to testing. [5]

The utility of the ACMG criteria will differ based on the population being studied. In populations with high consanguinity, homozygous recessive disorders are expected to predominate. Many of the autosomal recessive mutations such as $L R B A$ leading to CVID-like disorders have high penetrance, which may make application of the ACMG criteria more reliable. The high yield from consanguineous populations are a strong argument for routine genetic sequencing of affected individuals from these populations. [5] On the other hand, there will be fewer symptomatic patients (1:4) compared to those with autosomal dominant disorders (1:2) available for functional studies. In non-consanguineous populations, patients with genetic defects are more likely to be identified early if there is a family history of CVID or similar disorder.

In the event that no mutation relevant to the condition is identified, it is important for genetic findings to be periodically reviewed. Ideally NGS should comprise Whole Exome (or Genome) Sequencing (WES/WGS) with targeted analysis of results. This strategy would future-proof testing as new genes could be analysed from existing sequencing data when they are discovered. Gene panels rapidly become obsolete with the discovery of new genes causing the disorder.

There are now many commercial companies offering diagnostic NGS, either in the form of gene panels or WES with targeted analysis of the relevant genes. In some countries there are potential medico-legal implications from over or under-calling a variant as being pathogenic (causative). These companies do not have access to detailed clinical (phenotypic) data and are more likely to call a VUS. This can be frustrating for both patient and physician. Another potential issue is that companies may not report variants in genes they judge as not being relevant to the patient's disorder.

Study of the genetic basis of CVID and CVID-like disorders has highlighted the need for disease-specific bioinformatics specialists as well as close involvement of clinical experts caring for these patients. Currently commercial companies produce results and leave clinicians to deal with VUS. The ordering clinician, without the help of a bioinformatics specialist may not have the confidence to escalate such a VUS to being pathogenic (or benign) in spite detailed understanding of the patient's disease. Close collaboration between genetics professionals with disease-specific knowledge and the ordering clinician will increase the yield from genetic testing. CVID is perhaps the best exemplar why such collaboration is helpful, given the complexity of these conditions. It is also a strong argument for Clinical Immunology services to oversee genetic testing of their patients. 
In the longer term, we may see more precise quantitative tools, which not only analyse the individual variant, but also incorporate detailed clinical information in the decision tree. It is also possible large disease and gene specific databases and expert panels created in the future could assist with the interpretation of individual variants. Generating large disease-specific databases may prove more difficult for rare disorders but may become feasible when NGS becomes more widespread as a result of cost reduction. International collaborations under the aegis of the IUIS may allow the creation of such databases.

Such an approach, supported by clinical details represented in controlled vocabularies, may be well suited to artificial intelligence and machine learning, which could offer valuable assistance to clinicians and genetics professionals in determining the significance of an individual variant. With these measures, we hope that the frustrating scenario of VUS will become less frequent in the future. Initiatives such as the VUS List serve can provide interim assistance to clinicians dealing with these complexities.

We have previously discussed other disadvantages of genetic testing including genetic discrimination in the domains of employment and insurance.[5] Not all countries have enabling legislation such as the Americans with Disabilities Act (ADA) or the Genetic Information Non-discrimination Act (GINA). These disadvantages are not specific to CVID and CVID-like disorders but must be discussed at the time of genetic counselling and consent. Given their low yield and levels of uncertainty, we encourage our patients to opt out of disclosure of the ACMG "medically actionable genes". [32] VUS in these genes has the potential to cause great anxiety and expense if there is lack of insurance coverage in countries without a socialised health system.

In spite of the limitations outlined here, all future patients with a diagnosis of CVID should have genetic testing as part of their workup, after genetic counselling. Identifying CVID-like disorders has profound benefits to patients and their families. Those with CVID-like disorders caused by specific mutations now have new therapeutic options in addition to SCIG/IVIG.

\section{Establishing the diagnosis}

Confirming the clinical diagnosis of a CVID-like disorder

Identifying novel presentations of other CVID-like disorders eg as LOCID

Identifying atypical presentations of other PIDs with hypogammaglobulinemia eg XLP

Distinguishing genetic from acquired disorders eg drug-induced hypogammaglobulinemia Identifying digenic disorders THA

\section{Treatment}

Offering early SCIG/IVIG treatment for individuals carrying causative mutations Precision Medicine: specific treatment op Identifying patients who may benefit from gene based therapy in the future

\section{Prognosis}

Asymptomatic patients with monogenic defects have a high probability of symptomatic disease, leading to long-term SCIG/

\section{Pre-symptomatic testing}

Where presymptomatic diagnosis (at any age) is not possible with protein based tests eg patients with CVID-like disorders Diagnosis in infancy where conventional diagnostic tests are unreliable eg because of transplacentally acquired IgG levels

\section{Screening}

Cascade screening of at-risk relatives with or without symptoms after genetic counselling Identifying mutations from tissue

\section{PID prevention}

Prenatal diagnosis chorion villus sampling (CVS)

Pre-implantation genetic diagnosis (PGD)

\section{Research}

Characterising the role of molecules in cellular function

Assisting with the classification of primary immunodeficiency disorders

Identification of new genetic defects with trio analysis Investigating animal models of CVID-like disorders Identifying epista

Table 1. Advantages to identifying the underlying genetic defect in CVID-like disorders.[5] 


\section{ACMG guideline limitations}

- Can only be applied to monogenic defects

- Contradictory in silico analysis commonly encountered

- Databases may not have ethnic specific variants

- Pathogenic homozygous variants may be unidentified in healthy populations

\section{CVID- disease specific limitations}

- Variable penetrance and expressivity which can affect identification of de novo and segregation studies

- Presence of modifying or predisposing genes

- Cannot be applied to digenic or oligogenic disorders

- Cannot be applied to epigenetic disorders

- Late onset of symptomatic disease could affect de novo and segregation studies

- The presence of asymptomatic persons carrying the mutation

- Marked phenotypic variation- risk of misattribution of the mutation to a different disorder

- May not have access to functional studies for all affected genes

- Need for disease specific bioinformatics specialists

\section{Generic difficulties- issues common to other genetic conditions}

- Disputed paternity will affect segregation and de novo studies

- ACMG medically actionable genetic defects- low yield with risk of severe anxiety and great expense

\section{Generic issues relating to sequencing methodology}

\section{Sanger sequencing}

- Not practical given locus heterogeneity

- Problems identifying promoter mutations, complex rearrangements and pseudogenes

- May not identify intronic defects e.g. leading to cryptic splice sites

Whole exome sequencing

- Coverage issues leading to false positive or false negative results

- Problems identifying promoter mutations, complex rearrangements and pseudogenes

- May not identify intronic defects e.g. leading to cryptic splice sites

Whole genome sequencing

- More expensive than WES

- Has greater bioinformatics resource and interpretation challenges

Table 2 Summary of the limitations of the ACMG guidelines for CVID and CVID-like disorders

\section{References}

1. Abbott, J.K. and E.W. Gelfand, Common Variable Immunodeficiency : Diagnosis, Management, and Treatment. Immunology and Allergy Clinics of North America, 2015. 35 (4): p. 637-58.

2. Selenius, J.S., et al., Unexpectedly High Prevalence of Common Variable Immunodeficiency in Finland. Front Immunol, 2017. 8 : p. 1190.

3. Tseng, C.W., et al., The Incidence and Prevalence of Common Variable Immunodeficiency Disease in Taiwan, A Population-Based Study.PLoS One, 2015. 10 (10): p. e0140473.

4. Ameratunga, R., Assessing Disease Severity in Common Variable Immunodeficiency Disorders (CVID) and CVID-Like Disorders. Frontiers in Immunology, 2018. 9 (2130). 
5. Ameratunga, R., K. Lehnert, and S.T. Woon, All Patients With Common Variable Immunodeficiency Disorders (CVID) Should Be Routinely Offered Diagnostic Genetic Testing. Front Immunol, 2019. 10 : p. 2678 .

6. Ameratunga, R., D.M. Becroft, and W. Hunter, The simultaneous presentation of sarcoidosis and common variable immune deficiency.Pathology, 2000. 32 (4): p. 280-2.

7. Ameratunga, R. and S.T. Woon, Perspective: Evolving Concepts in the Diagnosis and Understanding of Common Variable Immunodeficiency Disorders (CVID). Clin Rev Allergy Immunol, 2019. (in press) .

8. Ameratunga, R., et al., Comparison of diagnostic criteria for Common Variable Immunodeficiency Disorder. Frontiers in Immunology, 2014. 5 : p. 415.

9. Ameratunga, R., et al., New diagnostic criteria for common variable immune deficiency (CVID), which may assist with decisions to treat with intravenous or subcutaneous immunoglobulin. Clin Exp Immunol, 2013. 174 (2): p. 203-11.

10. Ameratunga, R., et al., New diagnostic criteria for CVID.Expert Rev Clin Immunol, 2014. 10 (2): p. $183-6$.

11. Grimbacher, B., et al., Homozygous loss of ICOS is associated with adult-onset common variable immunodeficiency. Nat Immunol, 2003.4 (3): p. 261-8.

12. Schepp, J., et al., 14 Years after Discovery: Clinical Follow-up on 15 Patients with Inducible CoStimulator Deficiency. Front Immunol, 2017. 8 : p. 964.

13. Salzer, U., et al., Mutations in TNFRSF13B encoding TACI are associated with common variable immunodeficiency in humans. Nat Genet, 2005. 37 (8): p. 820-8.

14. Pan-Hammarstrom, Q., et al., Reexamining the role of TACI coding variants in common variable immunodeficiency and selective IgA deficiency. Nat Genet, 2007. 39 (4): p. 429-30.

15. Koopmans, W., et al., Clinical variability of family members with the C104R mutation in transmembrane activator and calcium modulator and cyclophilin ligand interactor (TACI). J Clin Immunol, 2013.33 (1): p. 68-73.

16. Ameratunga, R., et al., Epistatic interactions between mutations of TACI (TNFRSF13B) and TCF3 result in a severe primary immunodeficiency disorder and systemic lupus erythematosus. Clin Transl Immunology, 2017. 6 (10): p. e159.

17. Ameratunga, R., D. Gillis, and R. Steele, Diagnostic criteria for common variable immunodeficiency disorders. J Allergy Clin Immunol Pract, 2016. 4 (5): p. 1017-8.

18. Bonilla, F.A., et al., International Consensus Document (ICON): Common Variable Immunodeficiency Disorders. J Allergy Clin Immunol Pract, 2016. 4 (1): p. 38-59.

19. Seidel, M.G., et al., The European Society for Immunodeficiencies (ESID) Registry Working Definitions for the Clinical Diagnosis of Inborn Errors of Immunity. J Allergy Clin Immunol Pract, 2019.

20. Abolhassani, H., L. Hammarstrom, and C. Cunningham-Rundles, Current Genetic Landscape in Common Variable Immune Deficiency.Blood, 2020.

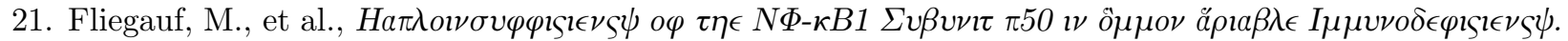
The American Journal of Human Genetics, 2015. 97 (3): p. 389-403.

22. Maffucci, P., et al., Genetic Diagnosis Using Whole Exome Sequencing in Common Variable Immunodeficiency. Front Immunol, 2016.7 : p. 220.

23. Ameratunga, R., et al., Primary immune deficiency disorders in the South Pacific: the clinical utility of a customized genetic testing program in New Zealand. Ann N Y Acad Sci, 2011. 1238 : p. 53-64. 
24. Ameratunga, R., et al., The clinical utility of molecular diagnostic testing for primary immune deficiency disorders: a case based review. Allergy Asthma Clin Immunol, 2010. 6 (1): p. 12.

25. Richards, S., et al., Standards and guidelines for the interpretation of sequence variants: a joint consensus recommendation of the American College of Medical Genetics and Genomics and the Association for Molecular Pathology. Genetics in medicine : official journal of the American College of Medical Genetics, 2015.17 (5): p. 405-24.

26. Strande, N.T., et al., Navigating the nuances of clinical sequence variant interpretation in Mendelian disease. Genet Med, 2018.20 (9): p. 918-926.

27. Gruber, C. and D. Bogunovic, Incomplete penetrance in primary immunodeficiency: a skeleton in the closet. Hum Genet, 2020.

28. Woon, S.T., et al., Follicular lymphoma in a X-linked lymphoproliferative syndrome carrier female. Scand J Immunol, 2008.68 (2): p. 153-8.

29. Ameratunga, R., et al., Keeping it in the family: the case for considering late onset combined immunodeficiency a subset of common variable immunodeficiency disorders. Expert Rev Clin Immunol, 2018.14 (7): p. 549-556.

30. Rodriguez-Cortez, V.C., et al., Monozygotic twins discordant for common variable immunodeficiency reveal impaired DNA demethylation during naive-to-memory B-cell transition. Nat Commun, 2015. 6 : p. 7335 .

31. Ameratunga, R., et al., Clinical Implications of Digenic inheritiance and epistasis in Primary Immunodeficiency Disorders. Front Immunol, 2018. 8 : p. 1965.

32. Haer-Wigman, L., et al., 1 in 38 individuals at risk of a dominant medically actionable disease. Eur J Hum Genet, 2019.27 (2): p. 325-330. 